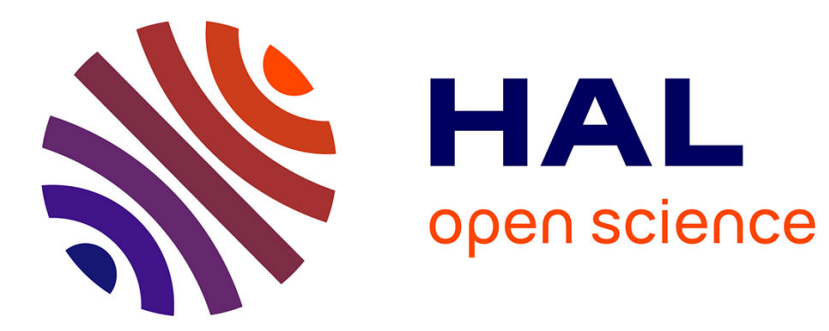

\title{
Enriching sparse mobility information in Call Detail Records
}

Guangshuo Chen, Sahar Hoteit, Aline Carneiro Viana, Marco Fiore, Carlos

Sarraute

\section{- To cite this version:}

Guangshuo Chen, Sahar Hoteit, Aline Carneiro Viana, Marco Fiore, Carlos Sarraute. Enriching sparse mobility information in Call Detail Records. Computer Communications, 2018, 122, pp.44-58. 10.1016/j.comcom.2018.03.012 . hal-01756120v2

\section{HAL Id: hal-01756120 \\ https://hal.science/hal-01756120v2}

Submitted on 3 Apr 2018

HAL is a multi-disciplinary open access archive for the deposit and dissemination of scientific research documents, whether they are published or not. The documents may come from teaching and research institutions in France or abroad, or from public or private research centers.
L'archive ouverte pluridisciplinaire HAL, est destinée au dépôt et à la diffusion de documents scientifiques de niveau recherche, publiés ou non, émanant des établissements d'enseignement et de recherche français ou étrangers, des laboratoires publics ou privés. 


\title{
Enriching Sparse Mobility Information in Call Detail Records
}

\author{
Guangshuo Chen ${ }^{\mathrm{a}, \mathrm{b}, *}$, Sahar Hoteit ${ }^{\mathrm{c}}$, Aline Carneiro Viana ${ }^{\mathrm{b}}$, Marco Fiore ${ }^{\mathrm{d}}$, \\ Carlos Sarraute \\ ${ }^{a}$ École Polytechnique, Université Paris Saclay, 91128 Palaiseau, France. \\ ${ }^{b}$ Inria, Université Paris Saclay, 91120 Palaiseau, France. \\ ${ }^{c}$ Laboratoire des Signaux et Systèmes, Université Paris Sud-CNRS-CentraleSupélec, \\ Université Paris-Saclay, 91192 Gif-sur-Yvette, France. \\ ${ }^{d}$ CNR-IEIIT, 10129 Torino, Italy. \\ ${ }^{e}$ Grandata Labs, 550 15th Street, San Francisco, 94103 California, USA
}

\begin{abstract}
Call Detail Records (CDR) are an important source of information in the study of diverse aspects of human mobility. The accuracy of mobility information granted by CDR strongly depends on the radio access infrastructure deployment and the frequency of interactions between mobile users and the network. As cellular network deployment is highly irregular and interaction frequencies are typically low, CDR are often characterized by spatial and temporal sparsity, which, in turn, can bias mobility analyses based on such data. In this paper, we precisely address this subject. First, we evaluate the spatial error in CDR, caused by approximating user positions with cell tower locations. Second, we assess the impact of the limited spatial and temporal granularity of CDR on the estimation of standard mobility metrics. Third, we propose novel and effective techniques to reduce temporal sparsity in CDR by leveraging regularity in human movement patterns. Tests with real-world datasets show that our solutions can reduce temporal sparsity in CDR by recovering $75 \%$ of daytime hours, while retaining a spatial accuracy within $1 \mathrm{~km}$ for $95 \%$ of the completed data.
\end{abstract}

Keywords: Call Detail Records, spatiotemporal trajectories, data sparsity,

\footnotetext{
* Corresponding author

Email addresses: guangshuo.chen@inria.fr (Guangshuo Chen), sahar.hoteit@u-psud.fr (Sahar Hoteit), aline.viana@inria.fr (Aline Carneiro Viana), marco.fiore@ieiit.cnr.it (Marco Fiore), charles@grandata.com (Carlos Sarraute)
} 
cellular networks, mobility, movement inference.

\section{Introduction}

Urbanization challenges the development and sustainability of city infrastructures in a variety of ways, and telecommunications networks are no exception. Understanding human habits becomes essential for managing the avail-

5 able resources in complex smart urban environments. Specifically, a number of network-related functions, such as paging [1], caching [2], dimensioning [3], or network-driven location-based recommending systems 4 have been shown to benefit from insights on movements of mobile network subscribers. More generally, the investigation of human mobility pattern has attracted a significant attention across disciplines [5] 9].

Motivation: Human mobility studies strongly rely on actual human footprints, which are usually provided by spatiotemporal datasets, as a piece of knowledge to investigate human mobility patterns. In this context, using specialized spatiotemporal datasets such as GPS logs seems to be a direct solution, but there is 15 a huge overhead of collecting such a detailed dataset at scale. Hence, Call Detail Records (CDR) have been lately considered as a primary source of data for large-scale mobility studies. CDR contain information about when, where and how a mobile network subscriber generates voice calls and text messages, and are collected by mobile network operators for billing purposes. These records usually cover large populations [10, which makes them a practical choice for performing large-scale human mobility analyses.

CDR can be regarded as footprints of individual mobility and can thus be used to infer visited locations, to learn recurrent movement patterns, and to measure mobility-related features. Despite the significant benefits that CDR bring to human mobility analyses, an indiscriminate use of CDR may question the validity of research conclusions. Indeed, CDR have limited accuracy in the spatial dimension (as the user's location is known at a cell sector or in a base station level) and the temporal dimension (since the device's position is only 
recorded when it sends or receives a voice call or text message). This is a severe limitation, as a cell (sector) typically spans thousands of square meters at least, and even a very active mobile network subscriber only generates a few tens of voice or text events per day. Overall, CDR are characterized by spatiotemporal sparsity, and understanding whether and to what extent such sparsity affects mobility studies is a critical issue.

35 Existing studies and limitations: A few previous works have investigated the validity of mobility studies based on CDR. An influential analysis 6 observed that using CDR allows to correctly identify popular locations that account for 90\% of each subscriber's activity; however, biases may arise when measuring individual human mobility features. Works such as [6] or the later [11 dis40 cussed biases introduced by the incompleteness of positioning information, i.e., the fact that CDR do not capture every location a user has travelled through. Nevertheless, another important bias of CDR, caused by the use of cell tower locations of mobile network subscribers in their footprints instead of their actual positions, has been overlooked in the literature.

Another open research problem is that of completing spatiotemporal gaps in CDR. The most intuitive solution is to consider that the location in an entry of CDR stays representative for a time interval period (e.g., one hour) centered on the actual event timestamp [7, 12]. So far and to the best of our knowledge, no more advanced solution has been proposed in the literature to fill the 50 spatiotemporal gaps in CDR.

Our work and contributions: In this paper, we explore the following research questions. First, we investigate how the spatiotemporal sparsity of CDR affects the accuracy and incompleteness of mobility information, by leveraging CDR and cell tower deployments in metropolitan areas. Second, we evaluate the biases caused by such spatiotemporal sparsity in identifying important locations and measuring individual movements. Third, we study the capability of CDR of locating a user continuously in time, i.e., the degree of completeness of the data. Answering these questions leads to the following main contributions: 
- We show that the geographical shifts, caused by the mapping of user locations to cell tower positions, are less than 1 kilometer in the most of cases (i.e., $85 \%-95 \%$ in the entire country or over $99 \%$ in the metropolitan areas in France), and the median of the shifts is around $200-500$ meters (varying across cellular operators). This result substantiates the validity of many large-scale analyses of human mobility that employ CDR.

- We provide a confirmation of previous findings in the literature regarding the capability of CDR to model individual movement patterns: (1) CDR provides the limited suitability for the assessment of the spread of human mobility and the study of short-term mobility patterns; (2) CDR yield enough details to detect significant locations in users' visiting patterns and to estimate the ranking among such locations.

- We implement different techniques for CDR completion proposed in the literature and assess their quality in the presence of ground-truth GPS data. Our evaluation sheds light on the quality of the results provided by each approach.

- We propose original CDR completion approaches that outperform existing ones, and carry out extensive tests on their performance with substantial real-world datasets collected by mobile network operators and mobility tracing initiatives. Validations against ground-truth movement information of individual users show that, on average, our proposed adaptive techniques can achieve an increased temporal completion of CDR data $(75 \%$ of daytime hours) and retain significant spatial accuracy (having errors below $1 \mathrm{~km}$ in $95 \%$ of completed time). Compared with the most common proposal in the literature, our best adaptive approach outperforms by $5 \%$ of accuracy and $50 \%$ of completion.

The rest of the paper is organized as follows. Related works are introduced in Sec.2 In Sec.3 we present the datasets used in our study. In Sec.4 we introduce and explore the biases of using CDR for human mobility analyses. 
In Sec.5, we discuss the rationale for CDR completion and errors introduced by common literature related approaches. In Sec.6 and 7 , we describe original CDR completion solutions that achieve improved accuracy, during nighttime and daytime, respectively. Finally, Sec.8 concludes the paper.

\section{Related works}

Our work aims at measuring and evaluating possible biases induced by the use of CDR. Understanding whether and to what extent these biases affect human mobility studies is a subject that has been only partly addressed. The early paper by Isaacman [13] unveiled that using CDR as positioning information may lead to a distance error within $1 \mathrm{~km}$ compared to ground-truth collected from 5 users. In a seminal work, Ranjan et al. 6] showed that CDR are capable of identifying important locations, but they can bias results when more complex mobility metrics are considered; the authors leveraged CDR of very active mobile network subscribers as ground-truth. In our previous study [14, we confirmed these observations using a GPS dataset encompassing 84 users. In the present work, we confirm the observation in [6], and push them one step further by also considering the spatial bias introduced by CDR. For the sake of completeness, we mention that results are instead more promising when mobility is constrained to transportation networks: Zhang et al. 11] found CDR-based individual trajectories to match reference information from public transport data, i.e., GPS logs of taxis and buses, as well as subway transit records.

Also relevant to our study are attempts at mitigating the spatiotemporal sparsity of CDR through completion techniques. The legacy approach in the literature consists in assuming that a user remains static from some time before and after each communication activity. The span of the static period, which we will refer to as temporal cell boundary hereinafter, is a constant system parameter that is often fairly arbitrary [12, 14]. In this paper, we extend previously proposed solutions [14, 15], and introduce two adaptive approaches to complete subscribers' trajectories inferred from CDR. 


\section{Datasets}

We leverage two types of datasets in our study. Coarse-grained datasets are typical CDR data and feature significant spatiotemporal sparsity as well as 681 million CDR in the selected period of study.

\footnotetext{
${ }^{1}$ Due to a non-disclosure agreement with the data owner, we cannot reveal the geographical area or the exact collecting period of this dataset.
} 


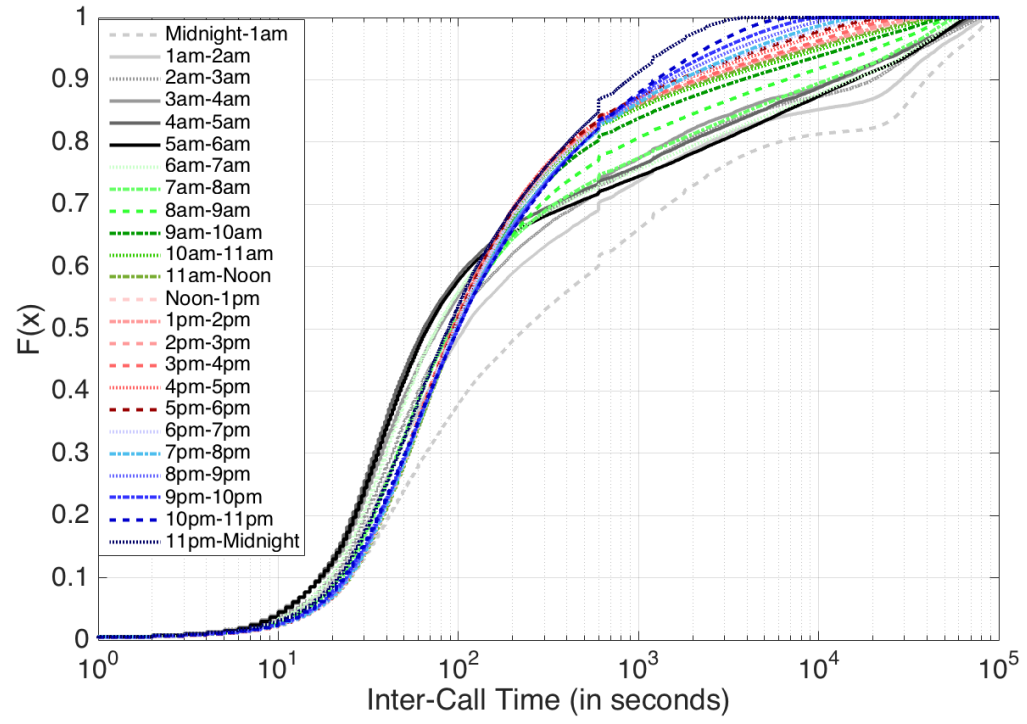

Figure 1: Distributions of the inter-event time in the CDR dataset at different day times.

We carry out a preliminary analysis of the CDR dataset, by extracting the experimental statistical distributions of the inter-event time (i.e., the time between consecutive events). These distributions will be later leveraged in Sec. 3.5 to downsample the fine-grained datasets. The resulting cumulative distribution functions (CDF) are shown, for different hours of the day, in Fig. 1. We observe that a majority of events occur at a temporal distance of a few minutes, but a non-negligible amount of events are spaced by hours. This observation confirms results in the literature on the burstiness of human digital communication activities, with rapidly occurring events separated by long periods of inactivity [16]. The curves in Fig. 1 allow appreciating the longer inter-event times during low-activity hours (e.g., midnight to $6 \mathrm{am}$ ) that become progressively shorter during the day.

\subsection{Internet flow fine-grained dataset}

This dataset is composed of mobile Internet session records, termed flows in the following. These records are generated and stored by the operator every time a mobile device establishes a TCP/UDP session for certain services 


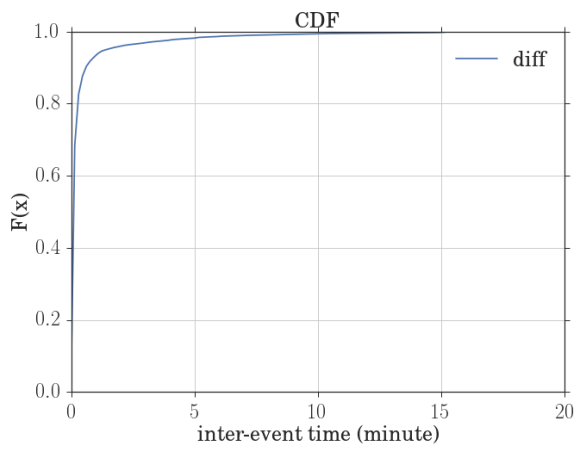

(a)

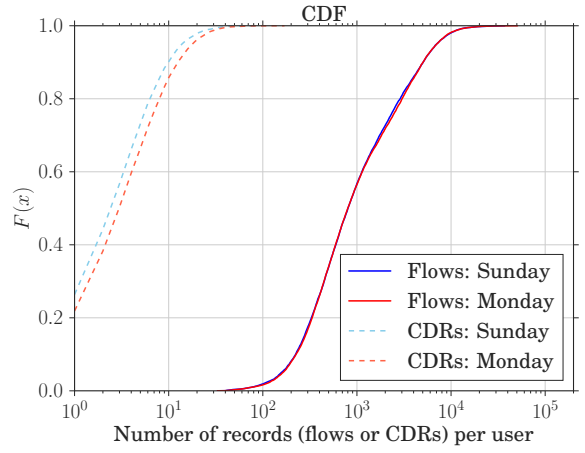

(b)

Figure 2: (a) CDF of the inter-event time in the Internet flow fine-grained dataset; (b) CDF of the number of records (flows or CDR) per user in a weekend and a weekday.

(i.e., Facebook, Google Services, WhatsApp etc). Each flow entry contains the

hashed device identifier, the type of service, the volume of exchanged upload and download data, the timestamps denoting the starting and ending time of the session, and the location of the cell tower handling the session. The dataset refers to two-day period consisting of a Sunday and a Monday in 2015. In each day, the data covers a constant time interval, i.e., from 10 am to $6 \mathrm{pm}$.

The flows in the Internet flow dataset have a considerably higher time granularity than the original CDR. Namely, at least one flow (i.e., one location) is provided within every 20 minutes, for all users. The statistical distribution of the per-user inter-flow time is shown in Fig.2(a). We note that in $98 \%$ of cases, the inter-event time is less than 5 minutes, and in less than $1 \%$ of cases, the inter-event time is higher than 10 minutes. We also plot in Fig.2(b) the CDF of the number of flows (solid lines) and CDR (dashed lines) for each user appearing in both datasets: the number of events per user in the Internet flow case is more than two orders of magnitude larger than that observed in the CDR case. We conclude that the Internet flows represent a suitable fine-grained dataset that can be associated to the coarse-grained CDR dataset.

Tab.1 summarizes the number of users in the Internet flow dataset. In particular, the over $10 \mathrm{~K}$ and $14 \mathrm{~K}$ subscribers recorded on Sunday and Monday, 
Table 1: Overview of the Internet Flow Dataset

\begin{tabular}{|c||c|c|c|}
\hline Day of the week & Users & Rare CDR users & Frequent CDR users \\
\hline Sunday & 10,856 & 6,154 & 4,702 \\
\hline Monday & 14,353 & 7,215 & 7,138 \\
\hline
\end{tabular}

respectively, are separated into two similarly sized categories based on their CDR as follows:

- Rare CDR users are not very active in placing or receiving voice calls and thus have limited records in the CDR dataset. As in [7, we use the threshold of 0.5 event/hour below which the user is considered to belong to this category.

- Frequent CDR users are more active callers or callees and have more than 0.5 event/hour in the CDR dataset.

This distinction will be leveraged later on in our performance evaluation.

\subsection{MACACO fine-grained dataset}

This dataset is obtained through an Android mobile phone application, MACACOApr2 developed in the context of the EU CHIST-ERA MACACO project [17. The application collects data related to the user's digital activities such as used mobile services, generated uplink/downlink traffic, available network connectivity, and visited GPS locations. These activities are logged with a fixed periodicity of 5 minutes. We remark that this sampling approach differs from those employed by popular GPS tracking projects, such as MIT Reality Mining [18] or GeoLife [19], where users' positions are sometimes irregularly sampled. With respect to such previous efforts, the regular sampling in MACACO data grants a neater and more comprehensive overview of a user's movement patterns. The MACACO data covers 84 users who have stayed in 6

\footnotetext{
${ }^{2}$ Available at https://macaco.inria.fr/MACACOApp/
} 
different countries and travel worldwide. The data collection spans 18 months

\subsection{Geolife fine-grained dataset}

This is the latest version of the Geolife dataset [19], which provides timestamped GPS locations of 182 individuals, mostly in Beijing [19]. The dataset spans a three-year time period, from April 2007 to August 2012. Unfortunately,

downsampling, we filter out users who have an insufficient number of records i.e., users with less than 30 records per day on average or less than 3 days 

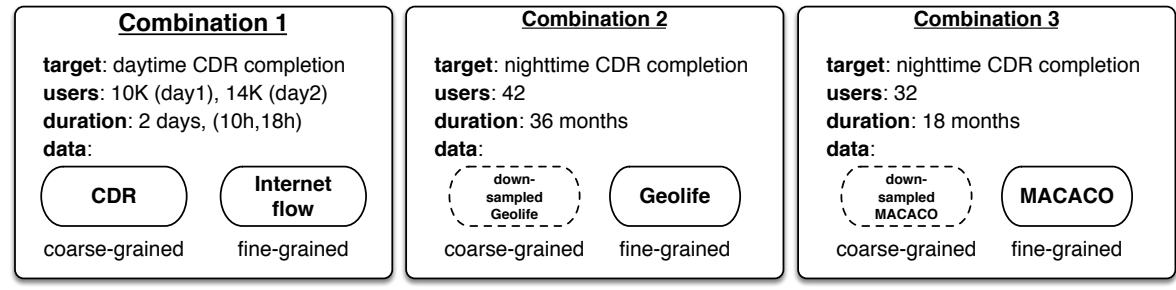

Figure 3: Combinations of corresponding coarse- and fine-grained datasets.

of activity. The final CDR-like coarse-grained versions of the MACACO and Geolife datasets contain 32 and 42 users, respectively.

By matching or downsampling the original data, we obtain three combinations of coarse-grained and fine-grained datasets for the same sets of users. Fig. 3 outlines them.

An important remark is that, as already mentioned in Sec.3.2, the Internet flow dataset only covers working hours, from 10 am to $6 \mathrm{pm}$. As a result, the first data combination is well suited to the investigation of CDR completion during daytime. The relevant analysis is presented in Sec.7.

The second and third data combinations, issued from the MACACO and Geolife datasets, cover instead all times. We thus employ them to overcome the limitations of the CDR and Internet flow pair, and to study CDR completion during night hours. Details are provided in Sec.6

\section{Biases in CDR-based mobility analyses}

Before delving into CDR completion, we present an updated analysis of the suitability of CDR data for the characterization of human mobility. Indeed, as anticipated in Sec. 1. CDR are typically sparse in space and time, which may affect the validity of results obtained from CDR mining. 


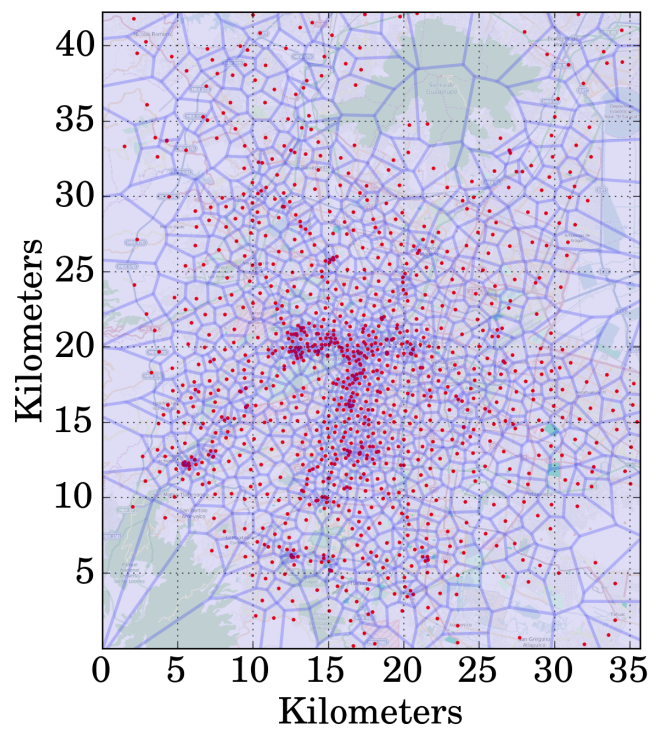

Figure 4: Deployment of cell towers in the target metropolitan area. Purple dots represent the base stations, whose coverage is approximated by a Voronoi tessellation.

\subsection{Cell tower locations}

In most CDR datasets, the position information is actually represented by the cell tower location handling the corresponding communication. Hence, a shift from the user's actual location to the cell tower location always exists in every CDR entry. Such a shift may impact the accuracy of individual mobility measurements. Usually, CDR are collected in metropolitan areas. In this case, the precision of human locations provided by CDR is related to the local deployment of base stations. Fig. 4 shows the deployment of cell towers in the metropolitan area where our CDR dataset was collected. The presence of cell towers is far from uniform, with a higher density in downtown areas where a cell tower covers an approximately $2 \mathrm{~km}^{2}$ area on average: in these cases, the cell coverage grants a fair granularity in the localization of mobile network subscribers. The same may not be true for cells in the city outskirts, which cover areas of several tens of $\mathrm{km}^{2}$.

We evaluate how the cell deployment can bias human mobility studies. To 


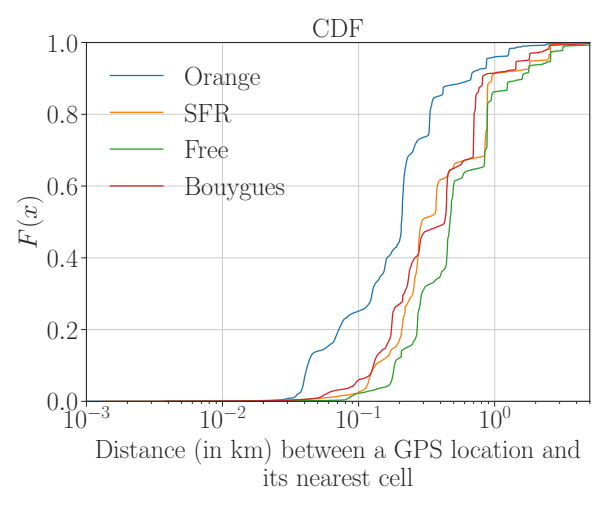

(a) Entire country

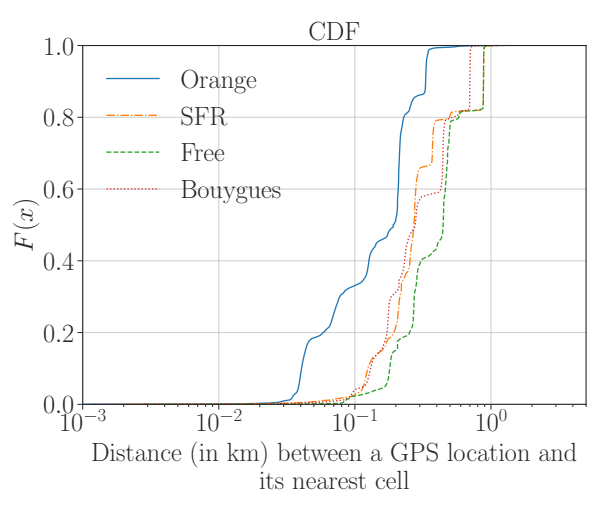

(b) Metropolitan areas

Figure 5: Distributions of the distances to the nearest cell tower (shifts), for 718, 987 GPS locations in the MACACO data of users in (a) the whole area and (b) major metropolitan areas (Paris Region, Lyon, Toulouse) in France.

this end, we perform a quantitative analysis of spatial shifts introduced by CDR positioning information by leveraging GPS logs in the MACACO dataset. Our focus on the MACACO dataset is due to two reasons: (i) the Internet flow and CDR datasets lack GPS information of visited locations or only provide cellular-level information of visited locations of their users; (ii) no available reliable source allows the extraction of cell tower information (i.e., coordinates or covered area of deployed cell towers) in the area of Beijing that the Geolife users are mainly from.

We first extract 718, 987 GPS locations in the mainland of France ${ }^{3}$ from the MACACO dataset. Among these locations, $74 \%$ are collected from the major metropolitan areas in France, including Paris Region, Lyon, and Toulouse. We then extract cell tower locations of the four major cellular network operators in France (i.e., Orange, SFR, Free, and Bouygues) from the open government data 21].

Fig. 5(a) is the CDF of the distance between each GPS location in the

\footnotetext{
${ }^{3}$ The study focuses on the area in the latitude and longitude ranges of $(43.005,49.554)$ and $(-1.318,5.999)$, respectively.
} 
MACACO dataset and its nearest cell tower. We observe that most of the locations have a distance below $1 \mathrm{~km}$ when shifting to their nearest cells (i.e., $95 \%$ for Orange, $91 \%$ for SFR, $86 \%$ for Free, and 91\% for Bouygues). Nevertheless, ing the geographical span of movement of individuals. For that, we compute for each user $u$ in the set of study $\mathcal{U}$ the radius of gyration, i.e., the deviation of the user's positions to their centroid. Formally, $R_{g}^{u}=\sqrt{\frac{1}{n} \sum_{i=1}^{n}\left\|\mathbf{r}_{i}^{u}-\mathbf{r}_{\text {centroid }}^{u}\right\|_{\text {geo }}^{2}}$, where $\mathbf{r}_{\text {centroid }}^{u}$ is the center of mass of locations of the user $u$, i.e., $\mathbf{r}_{\text {centroid }}^{u}=$ $300 \frac{1}{n} \sum_{i=1}^{n} \mathbf{r}_{i}^{u}$. This metric reflects how widely the subscribers move and is a popular measure used in human mobility studies [3, 5, 7, 23]. An individual who repeatedly moves among several fixed nearby locations still yields a small radius of gyration, even if she may total a large traveled distance.

We are able to compute both estimated (due to the temporal sparsity of the actual or the equivalent CDR data) and real (due to the finer granularity in the ground-truth provided by the Internet flow, MACACO, and Geolife datasets) 


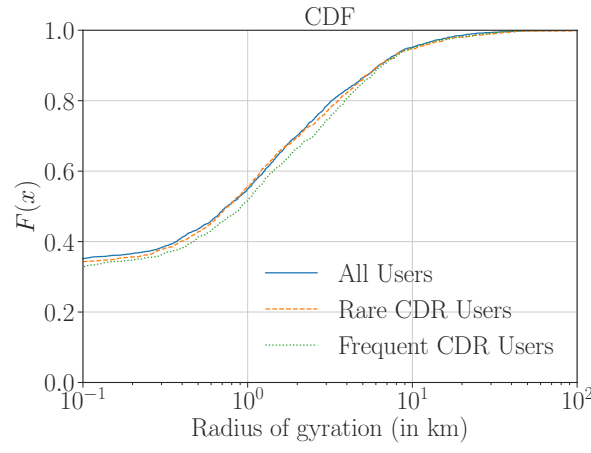

(a) Distribution

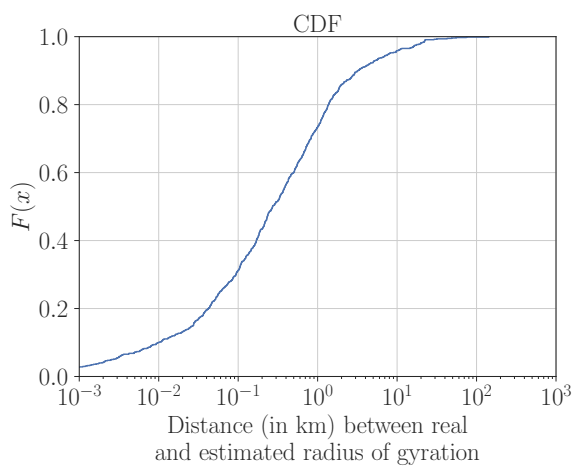

(c) Errors in MACACO data

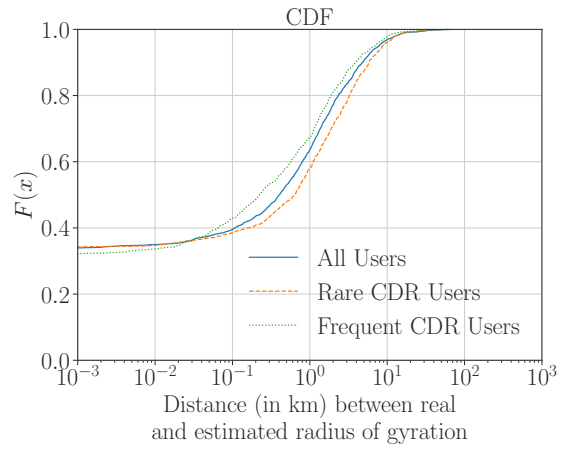

(b) Errors in Internet flow data

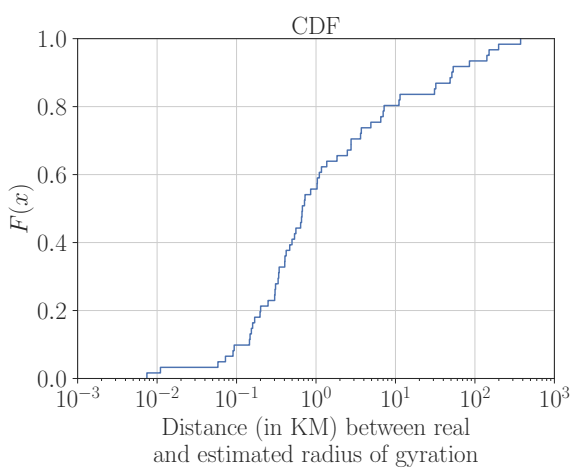

(d) Errors in Geolife data

Figure 6: (a) CDF of the radius of gyration of two categories (Rare and Frequent) of CDR users in the Internet flow dataset. (b)(c)(d) CDF of the distance between the real and the estimated radius of gyration from CDR over the users of the (b) Internet flow, (c) MACACO, and (d) Geolife datasets. 
radius of gyration for each user. Fig.6 summarizes the results.

Let us first consider the users of the Internet flow dataset and their radii of gyration. Three curves denote different cases: all, rare, and frequent CDR users (cf. Sec.3.2). The associated radius of gyration CDF are portrayed in Fig.6(a) The three distributions are quite similar, indicating that one can get a reliable distribution of $R_{g}^{u}$ from a certain number of users even if they are rare CDR users, i.e., have a limited number of mobile communication activities.

When considering the error between real and estimated radius of gyration, in Fig. 6(b) for the CDR and Internet flow datasets, and in Fig. 6(c) and 6(d) for the MACACO or Geolife datasets, respectively, we observe the following:

- The distribution of large errors is similar in all cases, and outlines a decent accuracy of the coarse-grained CDR or CDR-like datasets. For approximately $90 \%$ of the Internet flow users, $95 \%$ of the MACACO users and $70 \%$ of the Geolife users, the errors between the real and the estimated radius of gyration are less than $5 \mathrm{~km}$. The higher errors obtained from Geolife dataset may be interpreted by the irregular sampling in the original data and the presence of very large gaps between consecutive logs.

- A more accurate radius of gyration can be obtained for the CDR users who are especially active: $92 \%$ of the frequent CDR users have their errors lower than $5 \mathrm{~km}$, while the percentage decreases to $86 \%$ for the rare CDR users.

- When considering small errors, the distributions tend to differ, with far lower errors in the case of CDR than MACACO or Geolife. This is in fact an artifact of considering cell tower locations as the ground-truth user positions in the fine-grained Internet flow dataset ( $c f$. Sec.4.1). In the more accurate GPS data of MACACO and Geolife, around 30\% and 10\% of the users enjoy their errors lower than 100 meters, while around $35 \%$ of the users in the CDR dataset have errors below 1 meter.

Overall, these results confirm the previous findings on the limited suitability 
of CDR for the assessment of the spread of human mobility [6]. They also unveil how different datasets can affect the data reliability at diverse scales.

\subsection{Missing locations}

Due to spatiotemporal sparsity, the mobility information provided by CDR

CDR dataset, and plot in Fig. 7(a) the ratio $r_{N_{L}}$ of unique locations detected from CDR $\left(N_{L}^{\mathrm{CDR}}\right)$ to those from the ground-truth $\left(N_{L}^{\text {Flow }}\right)$, i.e., Internet flow data, as

$$
r_{N_{L}}=N_{L}^{\mathrm{CDR}} / N_{L}^{\text {Flow }}
$$

We notice that $42 \%$ in the population of study (i.e., all users) have their $r_{N_{L}}$ in the CDR data. The percentage of all users having this criterion is slightly higher for the frequent CDR users (50\%) and lower for the rare CDR users (37\%). These results confirm that using CDR to study very short-term mobility patterns is not a good idea due to the high temporal sparsity and the lack of

\subsection{Important locations}

The identification of significant places where people live and work is generally regarded as an important step in the characterization of human mobility. Here, we focus on home and work locations: we separate the period of study into two time windows, mapping to work time (9 am to $5 \mathrm{pm})$ and night time (10 pm to 7 am) for both CDR-like and ground-truth datasets. For each user, the places where the majority of work time records occur are considered a proxy for work locations; the equivalent records at night time are considered a proxy for home locations 24]. It is worth noting that, as the Internet flow dataset covers only $360(10 \mathrm{am}, 6 \mathrm{pm})$, we only infer work locations for this dataset.

Formally, let us consider a user $u$ from the user set. The visiting pattern of the user $u$ is a sequence of samples $\left\{\left(\ell_{u}^{1}, t_{u}^{1}\right), \ldots,\left(\ell_{u}^{n}, t_{u}^{n}\right)\right\}$, where the $i$-th sample $\left(\ell_{u}^{i}, t_{u}^{i}\right)$ denotes the location $\ell_{u}^{i}$ where the user $u$ is recorded at time $t_{u}^{i}$. The 


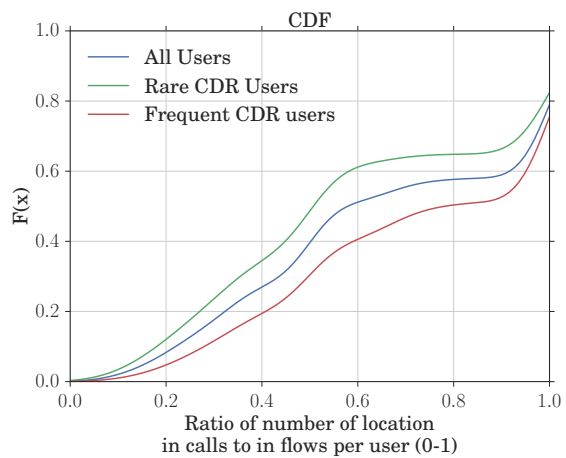

(a) Missing Ratio

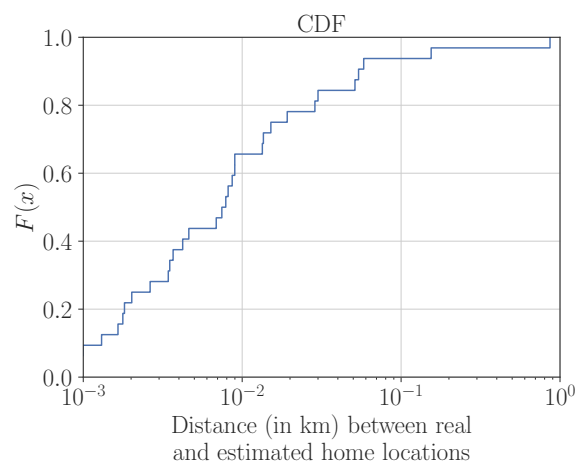

(c) Errors in MACACO data: Home

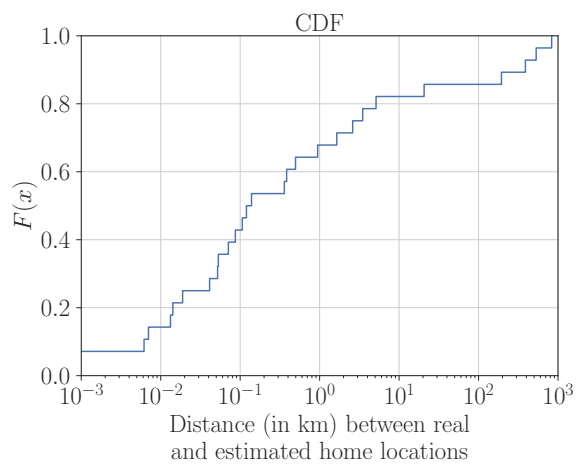

(e) Errors in Geolife data: Home

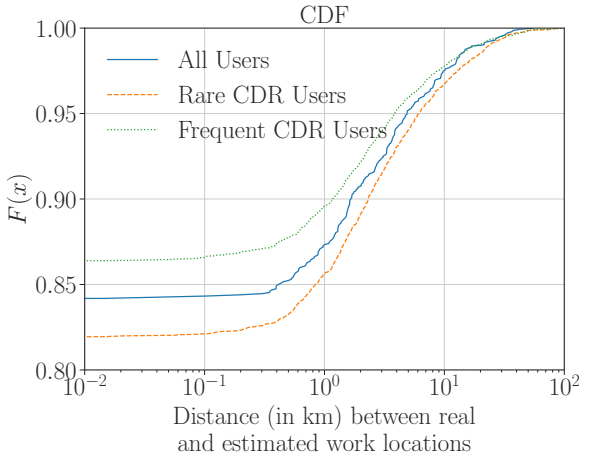

(b) Errors in Internet flow data: Work

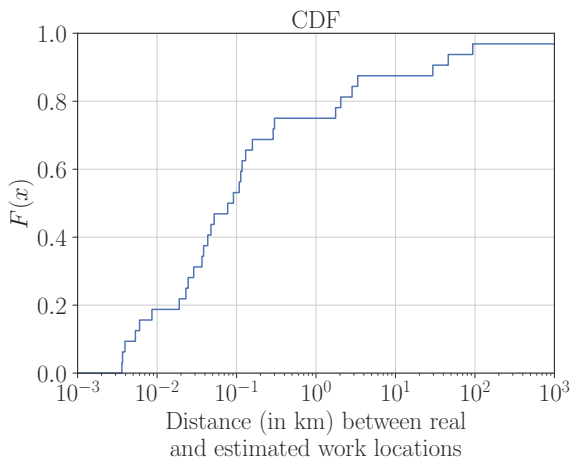

(d) Errors in MACACO data: Work

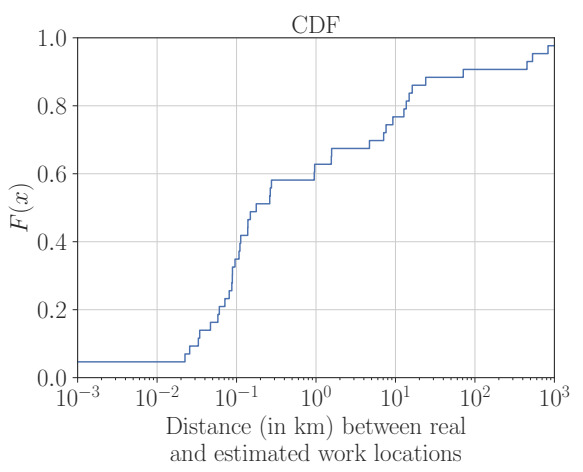

(f) Errors in Geolife data: Work

Figure 7: (a) CDF of the radio $r_{N_{L}}$ of the number of locations in each user's coarse-grained trajectory to the one in her fine-grained trajectory. (b)(c)(d)(e)(f) CDF of the distances between each user's real and estimated important locations located by her CDR and groundtruth: (b) work locations over the Internet flow users; (c) home and (d) work locations over the MACACO users; (e) home and (f) work locations over the Geolife users. 
home location $\ell_{u}^{H}$ of the user $u$ is then defined as the most frequent location

during night time:

$$
\ell_{u}^{H}=\operatorname{mode}\left(\ell_{u}^{i} \mid t_{u}^{i} \in t^{H}\right)
$$

where $t^{H}$ is the night time interval. The definition is equivalent for the work location $\ell_{u}^{W}$ of the user $u$, computed as

$$
\ell_{u}^{W}=\operatorname{mode}\left(\ell_{u}^{i} \mid t_{u}^{i} \in t^{W}\right)
$$

where $t^{W}$ is the work time interval.

We use the definitions in (2) and (3) to determine home and work locations and then evaluate the accuracy of the CDR-based significant locations by measuring the geographical distance that separates them from the equivalent locations estimated via the corresponding fine-grained ground-truth datasets.

The results are shown in Fig.7(b)-(f) as the CDF of the spatial error in the position of home and work places for different user groups for the three datasets. We observe the following:

- The errors related to home locations are fairly small in the MACACO dataset, but are relatively higher in the Geolife dataset. For the MACACO users, the errors are always below $1 \mathrm{~km}$ and $94 \%$ are within 100 meters. For the Geolife users, we observe that $17 \%$ of the errors are higher than $10 \mathrm{~km}$. A possible interpretation is that some Geolife users are highly active and don't stay within a stable location during nighttime.

- For both MACACO and Geolife users, the errors associated with work locations are sensibly higher than those measured for home locations. For instance, as shown in Fig. $7(\mathrm{~d})$, while $75 \%$ of the MACACO users have an error of less than 300 meters, the work places of a significant portion of individuals (around 12\%) are identified at a distance higher than $10 \mathrm{~km}$ from the positions extracted from the GPS data. A close behavior can be noticed from the Internet flow and Geolife users, as shown in Fig.7(b) and Fig. $7(\mathrm{f})$, These large errors typically occur for users who do not seem 
to have a stable work location and may be working in different places depending on, e.g., the time of day.

- The errors are significantly reduced when using cell tower locations as in the Internet flow dataset instead of actual GPS positions as in the MACACO or Geolife datasets. For the Internet flow users in Fig.7(b) the errors between the real and the estimated significant locations are null for approximately $85 \%$ of all users, indicating that the use of the coarsegrained dataset is fairly sufficient for inferring these significant locations.

- The errors are non-null for the remaining Internet flow users (15\%). Among them, $10 \%$ have relatively small errors (less than $5 \mathrm{~km}$ ), while $5 \%$ have errors larger than $5 \mathrm{~km}$.

- There is only a slight difference in the distribution of the errors associated with work locations between the rare and the frequent CDR users as shown in Fig.7(b), The reason is that, most of CDR are generated in significant locations, and hence the most frequent location obtained from CDR of a user is likely to be her actual work location during daytime. Still, it is relatively difficult to capture actual location frequencies if a user has only a few of CDR. Hence the rare CDR users have higher errors.

Overall, these results confirm previous findings [6], and further prove that CDR yield enough details to detect significant locations in users' visiting patterns. Besides, the results reveal a small possibility of incorrect estimation in the ranking among such locations.

\section{Current approaches to CDR completion}

The previous results confirm the quality of mobility information inferred from CDR, regarding the span of user's movement and significant locations. They also indicate that some biases are present: specifically, although transient and less important places visited may be lost in CDR data, capturing most of one's historical locations is not impossible. The good news is that, even in 
those cases, the error induced by CDR is relatively small. A major issue remains that CDR only provide instantaneous information about user's locations at a few time instants over a whole day. Overcoming the problem would help the already significant efforts in mobility analyses with CDR [10, allowing the exploration of scales much larger than those enabled by GPS datasets.

Temporal $C D R$ completion aims at filling the time gaps in CDR, by estimating users' locations in between their mobile communication activities. Several strategies for CDR completion have been proposed to date. In this section, we introduce and discuss the two most popular solutions adopted in the literature.

\subsection{Baseline static solution}

A simple solution is to hypothesize that a user remains static at the same location where she is last seen in her CDR. This methodology is adopted, e.g., by Khodabandelou et al. 25] to compute subscriber's presence in mobile traffic meta-data used for population density estimation. We will refer to this approach as the static solution and will use it as a basic benchmark for more advanced techniques. It is worth noting that this solution has no spatiotemporal flexibility; its performance only depends on the number of CDR a user generates in the period of study: i.e., the higher is the number of CDR, the lower will be the spatial error in the completed data by the static solution. In other words, there is no space (configurable setting or initial parameter) for customizing this solution to obtain better accuracy.

\subsection{Baseline stop-by solution}

Building on in-depth studies proving individuals to stay most of the time in the vicinity of their voice call places [26, Jo et al. 27] assume that users can be found at the locations where they generate some digital activities for an hour-long interval centered at the time when each activity is recorded. If the time between consecutive activities is shorter than one-hour, the inter-event interval is equally split between the two locations where the bounding events occur. This solution will be denoted as stop-by in the remaining sections. 


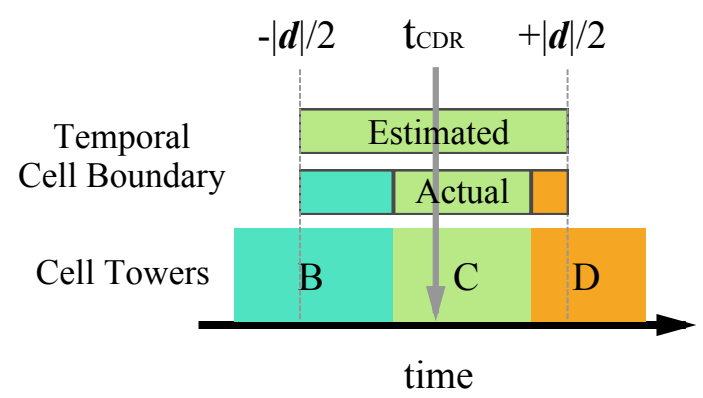

Figure 8: An example of a temporal cell boundary in the stop-by approach: A period $\left(t_{\mathrm{CDR}}-\right.$ $\left.|\mathbf{d}| / 2, t_{\mathrm{CDR}}+|\mathbf{d}| / 2\right)$ is given as a temporal cell boundary at the cell $C$ attached with a CDR entry at time $t_{\mathrm{CDR}}$. In this temporal cell boundary, the user is assumed to be at the cell $C$, while actually she moves from the cell $B$ to $D$ : this leads to a spatial error.

The drawback of the stop-by is that it uses a constant hour-long interval for all calls as well as users in CDR, which may be not always suitable. This solution lacks flexibility in dealing with various human mobility behaviors. As exemplified in Fig. 8, a single CDR is observed at time $t_{\mathrm{CDR}}$ at cell $C$. Following the stop-by solution, the user is considered to be stable at this cell $C$ during the period $\mathbf{d}=\left(t_{\mathrm{CDR}}-|\mathbf{d}| / 2, t_{\mathrm{CDR}}+|\mathbf{d}| / 2\right)$, while in fact the user has moved to two other cell towers during this period. We call the period estimated from an instant CDR entry, a temporal cell boundary. In the example of Fig. 8, this temporal cell boundary is overestimated.

Nevertheless, this solution has more flexibility than the static solution does, i.e., the time interval $|\mathbf{d}|$ affects its performance and is configurable. Although a one-hour interval ( $|\mathbf{d}|=60$ minutes) is usually adopted in the literature, we are interested in evaluating the performance of the stop-by solution over different intervals, which has never been explored before.

Intuitively, a spatial error occurs if the user moves to other different cells during the temporal cell boundary. To have a quantitative manner of such an error, we define the spatial error of a temporal cell boundary with a period $\mathbf{d}$ as follows:

$$
\operatorname{error}(\mathbf{d})=\frac{1}{|\mathbf{d}|} \int_{\mathbf{d}}\left\|c^{(\mathrm{CDR})}-c_{t}^{(\text {real })}\right\|_{\text {geo }} \mathrm{d} t .
$$



respectively. The high percentage of temporal cell boundaries with error $(\mathbf{d})=0$ in Fig.9 may be due to these static users, since they will not entail any spatial 


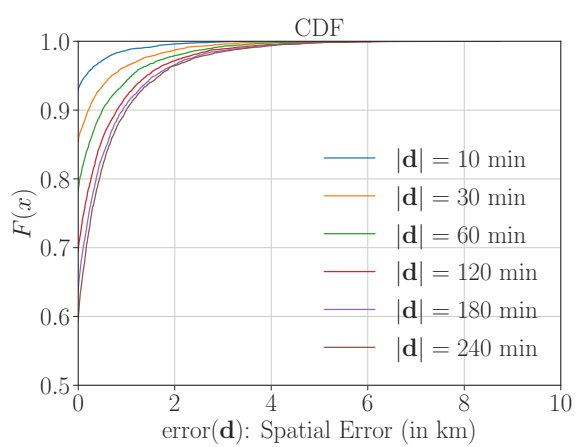

(a) CDF of Spatial Error: Monday

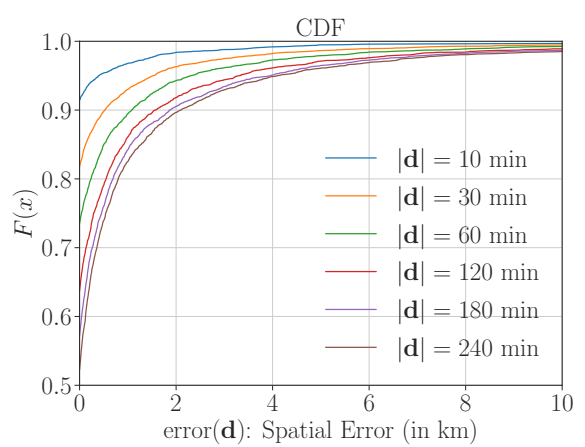

(b) CDF of Spatial Error: Sunday

Figure 9: CDF of the spatial error of temporal cell boundaries of CDR generated by the stop-by solution over two groups of the users in the Internet flow dataset on (a) Monday and (b) Sunday.

error, under any $|\mathbf{d}|$. To account for this aspect, we exclude the static users in the following, and only consider the mobile users, i.e., ones having $R_{g}^{u}>0$.

An interesting consideration is that the spatial error incurred by the stop-by approach is not uniform across cells. Intuitively, a cell tower covering a larger area is expected to determine longer user dwelling times and hence better estimates with stop-by. We thus compute for each cell its coverage as the cell radius: specifically, we assume a homogeneous propagation environment and an isotropic radiation of power in all directions at each cell tower, and roughly estimate each cell radius as that of the smallest circle encompassing the Voronoi polygon of the cell tower. We remark that this approach yields overlapping coverage at temporal cell boundaries, which reflects what happens in real-world deployments. In the target area under study, shown in Fig.4. $70 \%$ of the cells have radii within $3 \mathrm{~km}$, and the median radius is approximately $1 \mathrm{~km}$.

We can now evaluate the probability of having a temporal cell boundary with a null spatial error, as $P_{e 0}=\operatorname{Pr}\{\operatorname{error}(\mathbf{d})=0\}$. Fig.10(a) and 10(b) present the probabilities $P_{e 0}$ grouped by the cell radius, when applying varying sizes of temporal cell boundary on the days of study. We notice the following.

- The probability $P_{e 0}$ decreases with the increasing period marked by $|\mathbf{d}|$, 


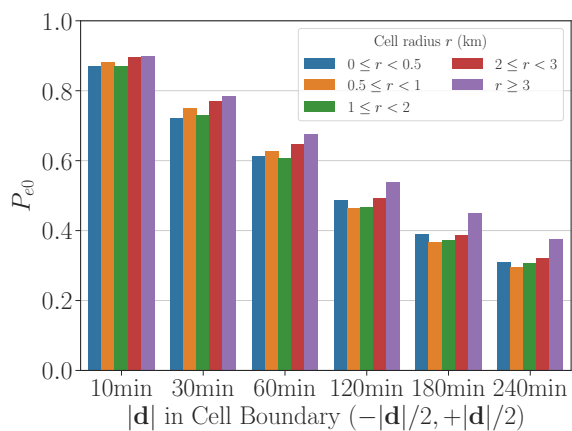

(a) $P_{e 0}$ : Monday

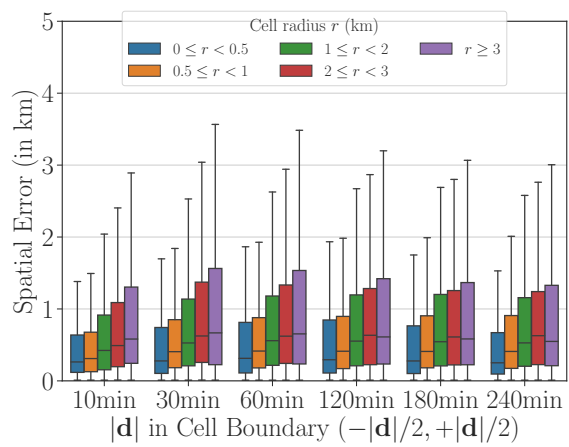

(c) Error: Monday

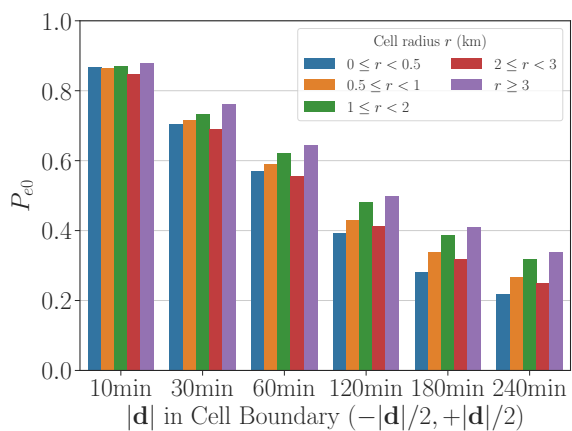

(b) $P_{e 0}$ : Sunday

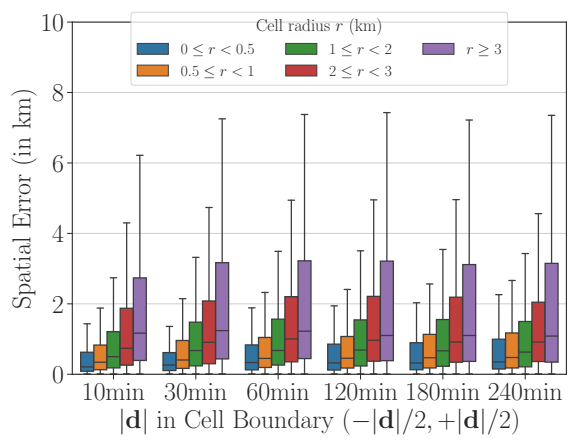

(d) Error: Sunday

Figure 10: Spatial errors of temporal cell boundaries of CDR generated by the stop-by solution over users with their $R_{g}>0$ : (a) (b) the probability $\left(P_{e 0}\right)$ of having a non-error temporal cell boundary $(-|\mathbf{d}|,|\mathbf{d}|)$, where $|\mathbf{d}| \in\{10,30,60,120,180,240\}$ minutes, under several groups of cell radius on (a) Monday and (b) Sunday; (c)(d) Box plot of non-zero spatial errors, grouped by the cell radius and the time period of temporal cell boundary on (c) Monday and (d) Sunday. Each box denotes the median and $25^{t h}-75^{t h}$ percentiles and the whiskers denote $5^{\text {th }}-95^{\text {th }}$ percentiles. 
indicating that using a large period on the temporal cell boundary increases the chances of generating some spatial errors. For instance, for $|\mathbf{d}|=30$ minutes, the probability of having a null spatial error is around 0.7 depending on the date and on the cell radius. When a larger $|\mathbf{d}|$ is used, the probability significantly increases (e.g., for $|\mathbf{d}|=60$ minutes, the probability $P_{e 0}$ reduces to around 0.6$)$.

- The probability $P_{e 0}$ correlates positively with the cell radius $r$. This trend is seen on both Monday and Sunday (except some cases), indicating that the cell size has an impact on the time interval during which the user stays within the cell coverage. Intuitively, handovers are frequent for users moving among small cells and less so for users traveling across large cells.

The results support the idea that there is a strong correlation between the temporal cell boundary and the cell coverage. Nevertheless, since CDR are usually sparse in time, using a small temporal cell boundary could only cover an insignificant amount of cell visiting time, while using a big temporal cell boundary increases the risk of having a non-null spatial error. To investigate this trade-off, we plot the variation of the statistical distribution of the spatial errors after excluding the null errors (i.e., keeping only cases with non-null error(d)) in Fig. 10(c) and 10(d) We observe that:

- The spatial error varies widely: it goes from less than $1 \mathrm{~km}$ to very huge values (up to $3.6 \mathrm{~km}$ on Monday and to $7.5 \mathrm{~km}$ on Sunday). Hence, for some users, the stop-by solution is unsuitable for reconstructing visiting patterns due to the presence of such high spatial errors.

- The spatial error grows with the cell radius: when the cell size increases, the variation of the error becomes wider, while the mean value also increases. This is reasonable because the higher the cell radius is, the farther the cell is from its cell neighbors. Hence, when a spatial error occurs, it means that the user is actually in a far cell that has a larger distance to 540 $c^{(\mathrm{CDR})}$. 


\subsection{Key insights}

Overall, we assert that temporal cell boundary estimates user's locations with a high accuracy when $|\mathbf{d}|$ is small. This validates the previous finding that users usually stay in proximity of call locations for certain time. The accuracy reduces significantly, giving rise to spatial errors, when increasing $|\mathbf{d}|$. Hence, the trade-off between the completion and the accuracy should be carefully considered when completing CDR using temporal cell boundaries. Using a constant $|\mathbf{d}|$ over all users as in the stop-by solution is unlikely to be an appropriate approach.

Building on these considerations, we propose enhancements to the stop-by and static solutions in the remainder of the paper. The data completion strategies introduced in the following leverage common trends in human mobility, in terms of (1) attachment to a specific location during night periods, and (2) a tendency to stay for some time in the vicinity of locations where digital activities take place. In particular, we tell apart strategies for CDR completion at night time and daytime: Sec.6 6 presents nighttime completion strategies inferring the home location of users; Sec.7 introduces our adaptive temporal cell boundary strategies leveraging human mobility regularity during daytime.

\section{Identifying temporal home boundaries}

The main goal of our strategies for CDR completion during nighttime is to infer temporal boundaries where users are located, with a high probability, at their home locations. We refer to this problem as the identification of the user's temporal home boundary. Gaps in CDR occurring within the home boundary of each user are then filled with the identified home location. The rationale for this approach stems from our previous observations that CDR allow identifying the home location of individuals with high accuracy.

\subsection{Proposed solutions}

We extend the stop-by solution ( $c f$. Sec. 5.2 in the following ways. Note that all techniques below assume that the home location is the user's most 
active location during some night time interval $\mathbf{h}$, and that CDR not in $\mathbf{h}$ are completed via legacy stop-by.

- The stop-by-home strategy adds fixed temporal home boundaries to the stop-by technique. If a user's location is unknown during $\mathbf{h}=(10 \mathrm{pm}, 7 \mathrm{am})$ due to the absence of CDR in that period, the user will be considered at her home location during $\mathbf{h}$.

- The stop-by-flexhome strategy refines the previous approach by exploiting the diversity in the habits of individuals. The fixed night time temporal home boundaries are relaxed and become flexible, which allows adapting them on a per-user basis. Specifically, instead of considering $\mathbf{h}=(10 \mathrm{pm}, 7 \mathrm{am})$ as the fixed home boundaries for all users, we compute for each user $u$ the most probable interval of time $\mathbf{h}_{\text {flex }}^{(u)} \subseteq \mathbf{h}$ during which the user is at her home location. Then, as for stop-by-home, the user will be considered at her home location to fill gaps in her CDR data during $\mathbf{h}_{\mathrm{flex}}^{(u)}$.

- The stop-by-spothome strategy augments the previous technique by accounting for positioning errors that can derive (1) from users who are far from home during some nights, or (2) from ping-pong effects in the association to base stations when the user is within their overlapping coverage region. In this approach, if a user's location during $\mathbf{h}_{\text {flex }}^{(u)}$ is not identified, and if she is last seen at no more than $1 \mathrm{~km}$ from her home location, she is considered to be at her home location.

We compare the above strategies with the static and the legacy stop-by solution introduced in Sec.5 assuming $|\mathbf{d}|=60 \mathrm{~min}$. Our evaluation considers dual perspectives. The first is accuracy, i.e., the spatial error between mobility metrics computed from ground-truth GPS data and from CDR completed with the different techniques above. The second is completion, i.e., the percent of the time during which the position of a user is determined. Note that the static solution (cf. Sec.5) provides user locations at all times, but this is not true for 
stop-by or the derived techniques above. In this case, the CDR is completed

only for a portion of the total period of study, and the users' whereabouts remain unknown in the remaining time.

\subsection{Accuracy and completion results}

We first compute the geographical distance between the positions in the GPS records in MACACO and Geolife and those in their equivalent CDR-like coarse-grained datasets. These strategies are not designed to provide positioning information at all times expect the static solution, hence distances are only measured for GPS samples whose timestamps fall in the time periods for which completed data is available.

Fig. 11(a) and 11(b) summarize the results of our comparative evaluation of accuracy, and allow drawing the following main conclusions:

- The static approach provides the worst accuracy in both datasets.

- The stop-by-flexhome technique largely improves the data precision, with an error that is lower than 100 meters in $90-92 \%$ of cases for the MACACO users and with a median error around 250 meters for the Geolife users.

- The stop-by-spothome technique provides the best performance for both datasets. For instance, about $95 \%$ of samples lie within 100 meters of the ground-truth locations in the MACACO dataset, while the median error is 250 meters (the lowest result) in the Geolife dataset.

These results confirm that a model where the user remains static for a limited temporal interval around each measurement timestamp is fairly reliable when it comes to accuracy of the completed data. They also support previous observations on the quite static behavior of mobile network subscribers [26]. More importantly, the information of home locations can be successfully included in such models, by accounting for the specificity of each user's habits overnight.

The stop-by and derived solutions do not provide full completion by design. Fig. 11(c) and 11(d) show the CDF of the hours per day during which a user 


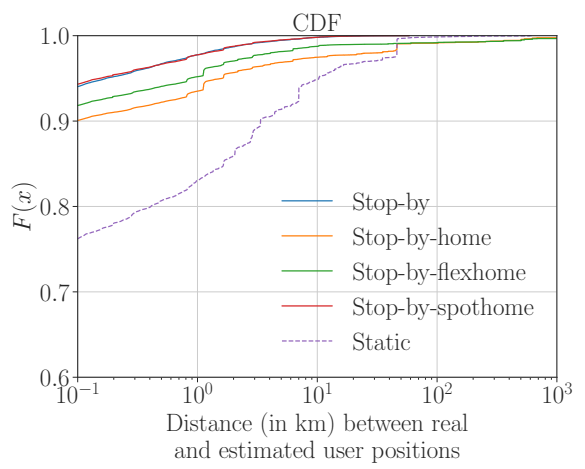

(a)

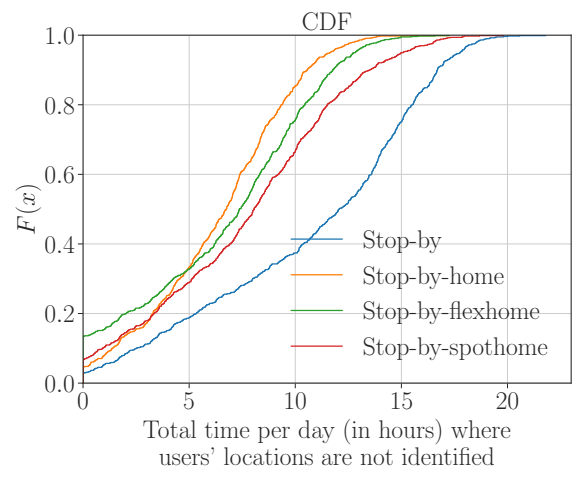

(c)

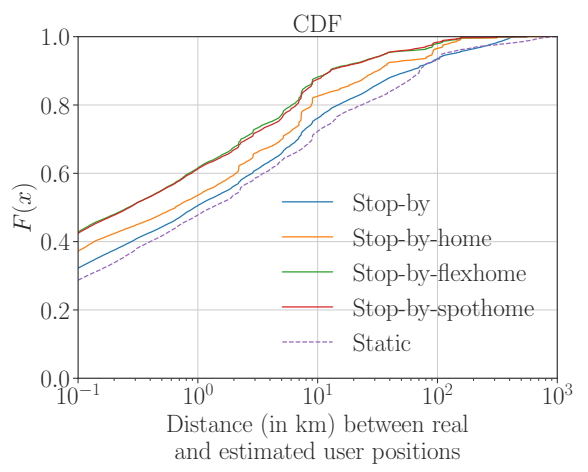

(b)

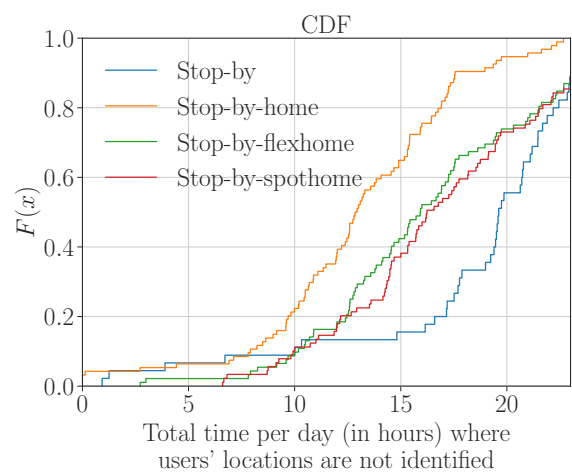

(d)

Figure 11: CDF of the spatial error (in $\mathrm{km}$ ) between samples from the GPS and completed data over the (a) MACACO and (b) Geolife data. CDF of the completion of completed data over the (c) MACACO and (d) Geolife data. 
cannot be localized by such solutions, for individuals in the MACACO and Geolife CDR-like datasets, respectively. The completion performance is in fact very heterogeneous across users, for all solutions: it can range from one hour per day for some individuals up to 23 hours per day for other subscribers. By comparing the plots, we assert that the more irregular sampling of the Geolife dataset translates into larger time gaps and smaller completion. Interestingly, the stop-by approach yields the worst result for both datasets, with unknown user positions in 12 and 19 hours per day in the median cases. Our proposed refinements to the stop-by solution increase the completion by inferring missing user positions overnight, when the CDR sampling is reduced. The improvement is significant, with a median gain over the basic stop-by solution of $4-5$ hours for MACACO dataset and 3-7 hours for the Geolife dataset.

Overall, the combination of the results in Fig. 11 indicates the stop-by-spothome solution as that achieving the best combination of high accuracy and fair completion, among the different completion techniques considered.

\section{Identifying temporal cell boundaries}

We now consider the possibility of completing CDR during daytime. Our strategy is based again on inferring temporal boundaries of users. However, unlike what has been done with nighttime periods in Sec.6, here we leverage the communication context of human mobility habits and extend the time span of the position associated with each communication activity to so-called temporal cell boundaries.

\subsection{Factors impacting temporal cell boundaries}

Hereafter, we aim to answer the following question: how to choose a proper and adaptive period for a temporal cell boundary instead of a static fixed-toall period? To answer the question, we need to understand the correlation between the routine behavior of users in terms of mobile communications and 
that can be extracted from CDR may affect daytime temporal cell boundaries. We categorize factors in three classes, i.e., event-related, long-term behavior, and location-related, as detailed next. Then, we leverage them to design novel approaches to estimate temporal cell boundaries.

\subsubsection{Event-related factors}

We include in this class the meta-data contained in records of common CDR datasets. They include the activity time, type (i.e., voice call or text message), and duration ${ }^{4}$ Intuitively, these factors have direct effects on temporal cell boundaries. For instance, in terms of time, a user may stay within a fixed cell during her whole working period. In terms of type and duration, a long phone call may imply that the user is static, while a single text message may indicate that the user is on the move. Besides, these factors are commonly found in and easily extracted from any common CDR entries.

\subsubsection{Long-term behavior factors}

This class includes factors describing users' activities over extended time intervals. They are the radius of gyration (URg), the number of unique visited locations (ULoc), and the number of active days during which at least one event is recorded (UDAY). These factors characterize a user by giving indications of ( $i$ ) her long-term mobility and (ii) her habit on generating calls and text messages, which may be indirectly related to her temporal cell boundaries. For each user, these factors are computed from our CDR dataset ( $c f$. Sec. 3.1) by aggregating data during the whole 3-month period of study.

\subsubsection{Location-related factors}

Factors in this class relate to positioning information. The first factor is the cell radius (CR), which we already proved to be affecting the reliability of CDR completion schemes in Sec.5. The other location-related factors take account for the relevance that different places have for each user's activities. The intuition is

\footnotetext{
${ }^{4}$ We set the duration text messages to 0 seconds.
} 
that individuals spend long time periods at their important places. Specifically, we explore it by applying the algorithm presented by Isaacman et al. [28, which determines prominent locations where the user usually spends a large amount of time or visits frequently.

The algorithm applies Hartigan's clustering [29] on visited cell locations of users in CDR and use logistic regression to estimate a location's importance to the user from factors extracted from the cluster that the location belongs to. To start with, the cluster approach chooses the cell tower from the first CDR and makes it the first cluster. Then, it recursively checks all cell towers in the remaining CDR. If a cell tower is within the distance threshold (we use $1 \mathrm{~km}$ ) to the centroid of a certain cluster, the cell tower is added to the cluster, and the centroid of the cluster is moved to the weighted average of the locations of all the cell towers in the cluster. The weights assigned to locations are the fractions of days in which they are visited over the whole observing period. The clustering process finishes once all cell towers are assigned to clusters.

Once clusters are defined, the importance of each cluster is identified according to the following observable factors: (i) the number of days on which any cell tower in the cluster was contacted (CDay); (ii) the number of days that elapse between the first and the last contact with any location in the cluster (CDur); (iii) the sum of the number of days cell towers in the cluster were contacted (CTDay); (iv) the number of cell towers inside the cluster (CTower); (v) the distance from the registered location of the activity to the centroid of the cluster 705 (CDist).

These factors derived from a cluster correlate with the time that the user spends in the cluster's locations, as shown by Isaacman et al. via their logistic regression model [28]. It is worth noting that we cannot reproduce the exact model in [28, since the used ground-truth is not publicly available. However, we can still use the same factors for our objective, i.e., identifying temporal cell boundaries. 


\subsection{Supervised temporal cell boundary estimation}

So far, we have introduced human behavior factors that might be directly or indirectly related to temporal cell boundaries. In order to use them for our purpose, we need a reliable model linking them to actual temporal cell boundaries. In the following we introduce two approaches to do so, both based on supervised machine learning.

\subsubsection{Symmetric and asymmetric temporal cell boundaries}

We define two kinds of temporal cell boundaries: symmetric and asymmetric. Given a CDR entry at time $t$, determining its temporal cell boundary means to expand the instantaneous time $t$ to a time interval $\mathbf{d}$, during which the user is assumed to remain within coverage of the same cell. For a symmetric temporal cell boundary, this period is generated from a CDR-based parameter $d^{ \pm}$as $\mathbf{d}=\left(t-d^{ \pm}, t+d^{ \pm}\right)$, i.e., it is symmetric with respect to the CDR time $t$. Instead, the period of an asymmetric temporal cell boundary is generated from two independent parameters $d^{+}$and $d^{-}$as $\mathbf{d}=\left(t-d^{-}, t+d^{+}\right)$.

We design sym-adaptive and asym-adaptive approaches, both of which receive a CDR entry as input and return an estimate of its associated temporal cell boundary. More precisely, the factors discussed in Sec.7.1 are extracted for each user and CDR record, and converted to an input vector $\mathbf{x}$, under the following rules: (i) the categorical factor type is converted to two binary features by one-hot encoding5? (ii) the time is converted to the distances (in seconds) separating it from 10am and from $6 \mathrm{pm}]^{6}$ (iii) the other factors are used as plain scalar values. Given a CDR entry and its input vector $\mathbf{x}$, we have the following approaches:

- The sym-adaptive approach contains one model that accepts the input vector and predicts the parameter $d^{ \pm}$as a symmetric estimation of the corresponding temporal cell boundary, i.e., $d^{ \pm}=F_{\mathrm{sym}}(\mathbf{x})$.

\footnotetext{
${ }^{5}$ Used to deal with the unbalanced occurrence of the types.

${ }^{6}$ Daytime interval covered by the used dataset ( $c f$. Sec. 3.2 .
} 
- The asym-adaptive approach contains two models that separately predict the parameters $d^{+}$and $d^{-}$as a joint asymmetric estimation of the corresponding temporal cell boundary, i.e., $d^{+}=F_{\text {asym }}^{+}(\mathbf{x})$ and $d^{-}=F_{\text {asym }}^{-}(\mathbf{x})$.

We use supervised machine learning techniques to build the models. It is worth noting that the user identifier is not in the input vector $\mathbf{x}$ because we do not want to train models that bound themselves to any particular user. This gives our models better flexibility and ensures higher potential for applying the trained model into other mobile phone datasets where the same factors can be derived.

\subsubsection{Estimating temporal cell boundaries via supervised learning}

We detail our methodology and results, by (i) formalizing the optimization problems that capture our goal, (ii) discussing how they can be addressed via supervised machine learning, and (iii) presenting a complete experimental evaluation.

Optimization problems. All the models are generalized from a training set $\mathcal{X}$ consisting of CDR entries (as input vectors) and their real temporal cell boundaries (which are originally asymmetric), i.e., $\mathcal{X}=\left\{\left(\mathbf{x}_{i}, d_{i}^{+}, d_{i}^{-}\right)\right\}$.

To build the asym-adaptive approach, the objective is to find two separate approximations, as $F_{\text {asym }}^{+}(\mathbf{x})$ and $F_{\text {asym }}^{-}(\mathbf{x})$, to functions $F^{+}(\mathbf{x})$ and $F^{-}(\mathbf{x})$ that respectively minimize the expected values of two losses $L\left(d^{+}, F^{+}(\mathbf{x})\right)$ and $L\left(d^{-}, F^{-}(\mathbf{x})\right)$, i.e.,

$$
\begin{aligned}
& F_{\text {asym }}^{+}(\mathbf{x})=\arg \min _{F^{+}} \mathbb{E}_{d^{+}, \mathbf{x}}\left[L\left(d^{+}, F^{+}(\mathbf{x})\right)\right], \\
& F_{\text {asym }}^{-}(\mathbf{x})=\arg \min _{F^{-}} \mathbb{E}_{d^{-}, \mathbf{x}}\left[L\left(d^{-}, F^{-}(\mathbf{x})\right)\right],
\end{aligned}
$$

where $L$ is the squared error loss function, i.e., $L(x, y)=\frac{1}{2}(x-y)^{2}$.

To build the sym-adaptive approach, a modified training set $\mathcal{X}^{ \pm}=\left\{\left(\mathbf{x}_{i}, d_{i}^{ \pm}\right)\right\}$ is firstly generated from the original $\mathcal{X}$ by applying $d_{i}^{ \pm}=\min \left\{d_{i}^{+}, d_{i}^{-}\right\}$on each real asymmetric temporal cell boundary. Then, as our objective, we need to find an approximation $F_{\mathrm{sym}}(\mathbf{x})$ to a function $F^{ \pm}(\mathbf{x})$ that minimizes the expected 
value of the $\operatorname{loss} L\left(d^{ \pm}, F^{ \pm}(\mathbf{x})\right)$, i.e.,

$$
F_{\text {sym }}(\mathbf{x})=\underset{F^{ \pm}}{\arg \min } \mathbb{E}_{d^{ \pm}, \mathbf{x}}\left[L\left(d^{ \pm}, F^{ \pm}(\mathbf{x})\right)\right] .
$$

cross validation and discuss later. In the asym-adaptive approach, the same algorithm is used except that the training set $\mathcal{X}^{ \pm}$is replaced by $\mathcal{X}$. 


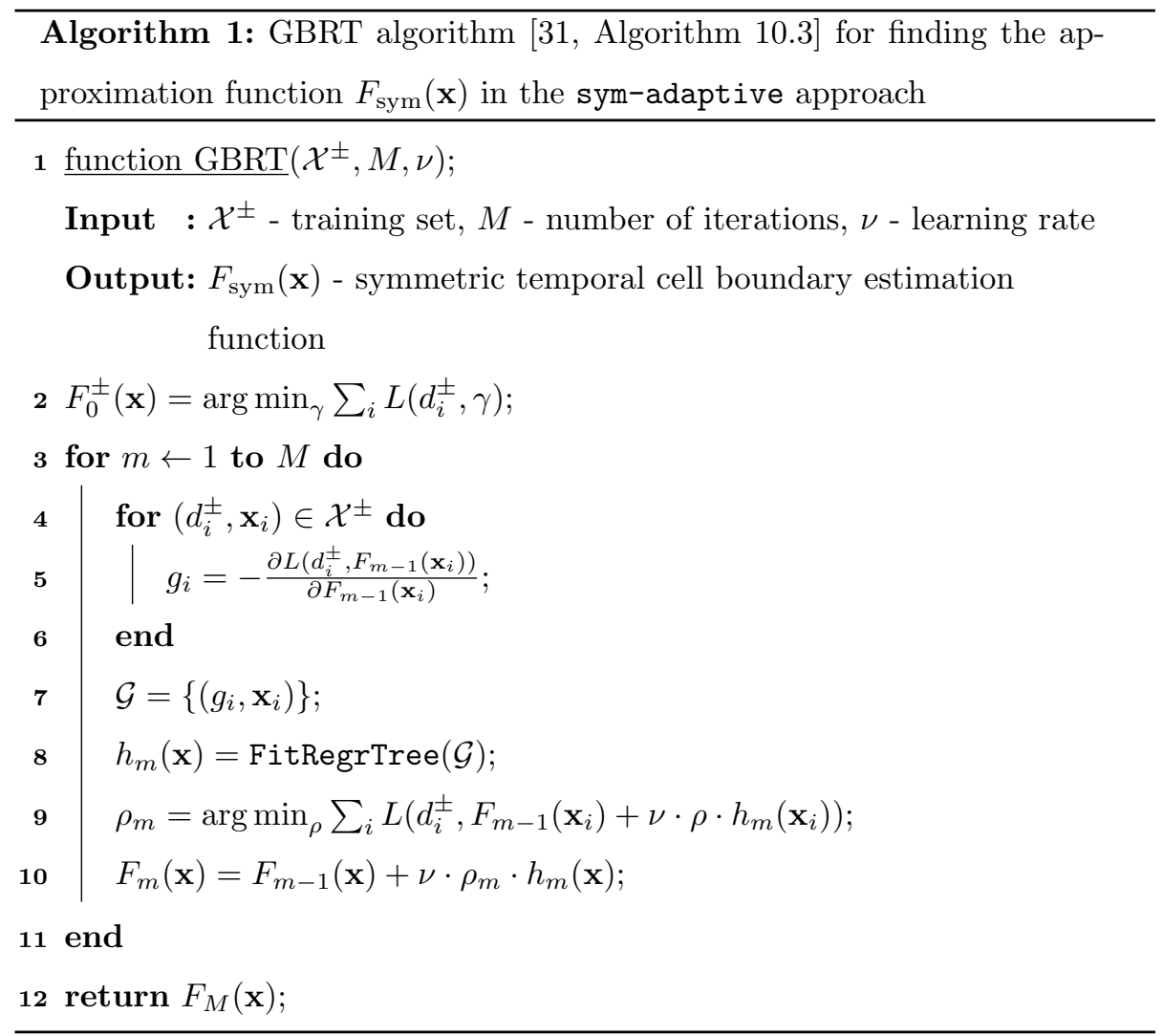

Experiments. The first step is to build the training sets. For that, we randomly select $50 \%$ of the users from the two available days (i.e., a Monday and a Sunday) in the Internet flow dataset ( $c f$. Sec. 3.2). In particular, from the CDR and Internet flow datasets, we first extract for each CDR entry of these selected users its corresponding input vector $\mathbf{x}$ as well as the parameters $d^{+}, d^{-}$of its real temporal cell boundary. We then build the two training sets $\mathcal{X}$ and $\mathcal{X}^{ \pm}$.

The second step is to build the approximation functions (i.e., $F_{\text {asym }}^{+}, F_{\text {asym }}^{-}$, and $\left.F_{\text {sym }}\right)$ from the training sets. For that, we have to first tune the $M$ and $\nu$ parameters of Alg. 1 of each approximation function. To this end, we use a 3 -fold cross-validation to select the number of iterations $M$ from the candidate set $\{100,500,1000,2000, \cdots, 10000\}$ and the learning rate $\nu$ from the candidate set $\{0.1,0.2, \cdots, 1\}$. In particular, we divide the training set $\mathcal{X}$ (or $\mathcal{X}^{ \pm}$) into 


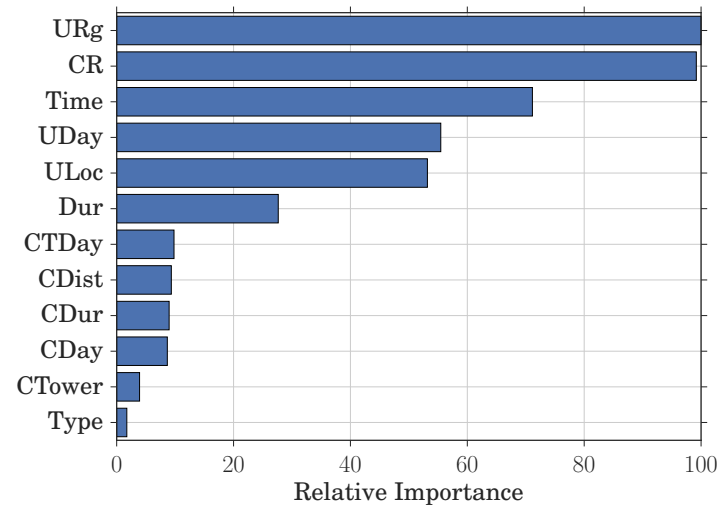

Figure 12: Relative Importance of features in determining accurate temporal cell boundaries.

equal-sized three subsets. For each combination of $M$ and $\nu$, we train the model corresponding to each approximation function based on one subset and validate it on the other two subsets. We repeat this operation three times with each of the subsets used as training data. We select as our actual parameters the $M$ and $\nu$ values that achieve the lowest loss in the cross-validation. Finally, we use the training sets $\mathcal{X}$ and $\mathcal{X}^{ \pm}$and the tuning parameters that we select to build the functions $F_{\text {asym }}^{+}, F_{\text {asym }}^{-}$, and $F_{\text {sym }}$ corresponding to the asym-adaptive and sym-adaptive approaches.

Fig.12 shows the relative importance of factors with respect to the estimation of a temporal cell boundary in the training procedure of the GBRT technique. For each factor, its importance is computed as a relative value of the sum of its corresponding importance in all the three approximations. The importance indicates the degree of a feature contributing to the construction of the regression trees. This figure allows us drawing the following main conclusions, valid for both approaches.

- The three most important factors are the timestamp of the activity, the cell radius, and the radius of gyration. This indicates that the time spent by a user within coverage of a same cell mainly depends on the cell size, the precise time when the activity occurred, and the user's long-term mobility. 


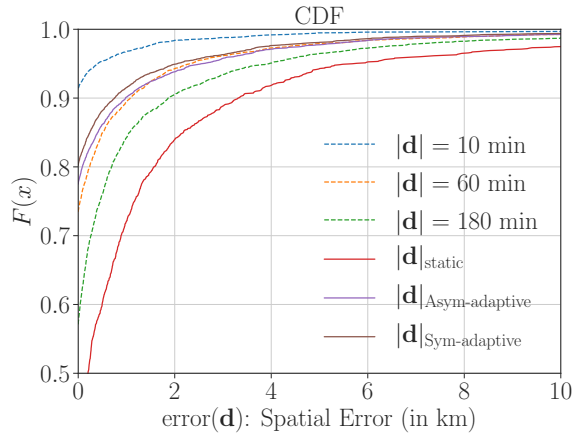

(a) Spatial Error: Sunday

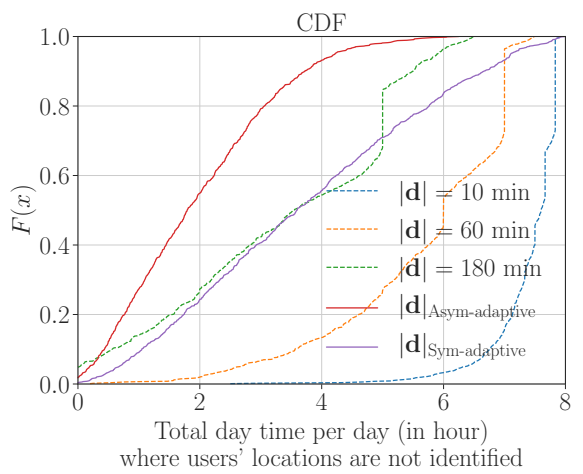

(c) Completion: Sunday

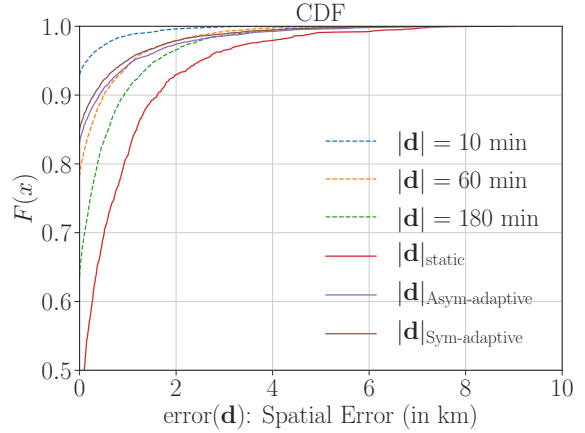

(b) Spatial Error: Monday

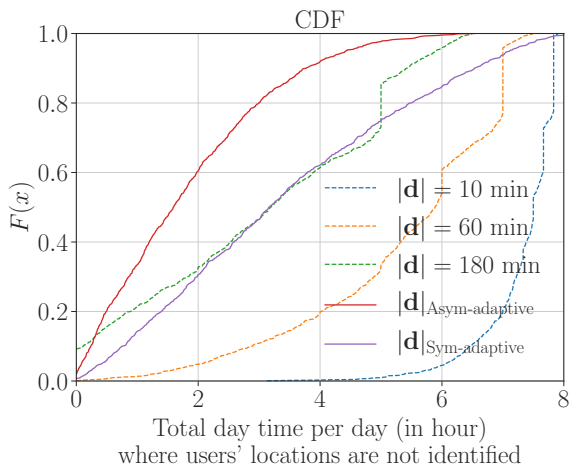

(d) Completion: Monday

Figure 13: CDF of the spatial errors of temporal cell boundaries computed on (a) Sunday and (b) Monday; CDF of the completion of completed data on (c) Sunday and (d) Monday, across the stop-by, static, sym-adaptive, and asym-adaptive approaches.

- Surprisingly, the activity's type is the least relevant factor, indicating that knowing whether a user generates a call or a message is useless in determining a temporal cell boundary.

\subsection{Accuracy and completion results}

We compare our two trained approaches with the stop-by and static approaches using the CDR from the remaining $50 \%$ of the randomly-selected users. For the two sym-adaptive and asym-adaptive approaches, we build two testing sets from the CDR entries of the remaining users. We then let them generate 
adaptive symmetric and asymmetric temporal cell boundaries using the input vectors in the testing sets. Besides, we let the stop-by approach generate temporal cell boundaries using $|\mathbf{d}|=\{10,60,180\}$ minutes. As in Sec. 6, we make a comparative study by evaluating the solutions regarding accuracy and completion, where the accuracy is measured by evaluating the spatial error in (4) (cf. Sec.5). Recall that a good data completion approach should cover the observing period as much and precise as possible, i.e., satisfying high accuracy and completion simultaneously.

Fig.13(a) and 13(b) display the distribution of the spatial errors over all temporal cell boundaries. Our results confirm that the spatial error increases as $t_{d}$ becomes larger when using the stop-by approach. More importantly, the two adaptive approaches perform slightly better than the stop-by approach does with its most common setting $(|\mathbf{d}|=60$ minutes) in terms of the spatial error. As expected, the static solution has the worst performance, similarly to what observed in the case of home boundaries using the MACACO and Geolife datasets.

Fig. 13(c) and 13(d) plot the distribution of the completion per users over all approaches except static (of which the completed data always covers the whole period). The $\mathrm{x}$-axis of the figures has 8 hours because the Internet flow dataset only covers an eight-hour day time, i.e., $(10 \mathrm{am}, 6 \mathrm{pm})$. We remark that both our adaptive approaches score a significant performance improvement in terms of completion: the amount of time during which users' locations stay unidentified is substantially reduced with respect to the legacy stop-by approach. On average, only approximately 2 hours ( $25 \%$ of the period of study) of the user's day time remains unidentified after applying the asym-adaptive approach, while 3 hours remains unidentified after using the sym-adaptive and stop-by $(|\mathbf{d}|=180$ minutes) approaches. The stop-by approach with its most common setting $(|\mathbf{d}|=60$ minutes $)$ has the same degree of accuracy as the adaptive approaches but has a far less degree of completion (i.e., a median of 6 unidentified hours).

Overall, these results highlight a clear advantage provided by adaptive approaches for CDR completion based on supervised learning. Consequently, the 
adaptive approaches achieve a slightly better performance in terms of accuracy but have a far better performance in terms of completion. The asym-adaptive approach has an obvious advantage than the competitors: it completes $75 \%$ of the day hours with a fairly good accuracy.

\section{Conclusion}

In this paper, we leveraged real-world CDR and GPS datasets to characterize the bias induced by the use of CDR for the study of human mobility, and

\section{Acknowledgment}

This work is supported by the EU FP7 ERANET program under grant CHIST-ERA-2012 MACACO and is performed in the context of the EMBRACE Associated Team of Inria. 


\section{References}

890

[1] H. Zang, J. C. Bolot, Mining call and mobility data to improve paging efficiency in cellular networks, in: MobiCom '07: Proceedings of the 13th annual ACM international conference on Mobile computing and networking, ACM, New York, New York, USA, 2007, pp. 123-134.

[2] K. Y. Lai, Z. Tari, P. Bertok, Supporting user mobility through cache relocation, Mobile Information Systems 1 (4) (2005) 275-307.

[3] U. Paul, A. P. Subramanian, M. M. Buddhikot, S. R. Das, Understanding traffic dynamics in cellular data networks, in: INFOCOM, 2011 Proceedings IEEE, IEEE, 2011, pp. 882-890.

[4] Y. Zheng, L. Zhang, X. Xie, W.-Y. Ma, Mining interesting locations and travel sequences from gps trajectories, in: Proceedings of the 18th international conference on World wide web, ACM, 2009, pp. 791-800.

[5] M. C. González, C. A. Hidalgo, A.-L. Barabási, Understanding individual human mobility patterns, Nature 453 (7196) (2008) 779-782.

[6] G. Ranjan, H. Zang, Z.-L. Zhang, J. Bolot, Are call detail records biased for sampling human mobility?, SIGMOBILE Mob. Comput. Commun. Rev. 16 (3) (2012) 33-44. doi:10.1145/2412096.2412101.

[7] C. Song, Z. Qu, N. Blumm, A.-L. Barabási, Limits of Predictability in Human Mobility, Science 327 (5968) (2010) 1018-1021.

[8] C. Iovan, A.-M. O. Raimond, T. Couronné, Z. Smoreda, Moving and Calling: Mobile Phone Data Quality Measurements and Spatiotemporal Uncertainty in Human Mobility Studies., AGILE Conf. (Chapter 14) (2013) $247-265$.

[9] M. Ficek, L. Kencl, Inter-Call Mobility model: A spatio-temporal refinement of Call Data Records using a Gaussian mixture model., IEEE INFOCOM 2012 (2012) 469-477. 
[10] D. Naboulsi, M. Fiore, S. Ribot, R. Stanica, Large-scale Mobile Traffic Analysis: a Survey, IEEE Communications Surveys \& Tutorials PP (99) (2015) 1-1.

[11] D. Zhang, J. Huang, Y. Li, F. Zhang, C. Xu, T. He, Exploring human mobility with multi-source data at extremely large metropolitan scales, in: Proc. of MobiCom, New York, USA, 2014. doi:10.1145/2639108. 2639116

[12] H. H. Jo, M. Karsai, J. Karikoski, K. Kaski, Spatiotemporal correlations of handset-based service usages, EPJ Data Science 1 (2012) 1-18.

[13] S. Isaacman, R. Becker, R. Cáceres, S. Kobourov, M. Martonosi, J. Rowland, A. Varshavsky, Ranges of human mobility in los angeles and new york, in: Pervasive Computing and Communications Workshops (PERCOM Workshops), 2011 IEEE International Conference on, IEEE, 2011, pp. 88-93.

[14] S. Hoteit, G. Chen, A. Viana, M. Fiore, Filling the gaps: On the completion of sparse call detail records for mobility analysis, in: Proceedings of the Eleventh ACM Workshop on Challenged Networks, CHANTS '16, ACM, New York, NY, USA, 2016, pp. 45-50. doi:10.1145/2979683.2979685

[15] G. Chen, A. C. Viana, C. Sarraute, Towards an adaptive completion of sparse call detail records for mobility analysis, in: Pervasive Computing and Communications Workshops (PerCom Workshops), 2017 IEEE International Conference on, IEEE, 2017, pp. 302-305.

[16] A.-L. Barabasi, The origin of bursts and heavy tails in human dynamics, Nature 435 (2005) 207.

[17] EU CHIST-ERA Mobile context-Adaptive CAching for COntent-centric networking (MACACO) project, https://macaco.inria.fr/. 
[18] N. Eagle, A. (Sandy) Pentland, Reality mining: Sensing complex social systems, Personal Ubiquitous Comput. 10 (4) (2006) 255-268. doi:10. 1007/s00779-005-0046-3

[26] M. Ficek, L. Kencl, Inter-call mobility model: A spatio-temporal refinement of call data records using a gaussian mixture model, in: INFOCOM, 2012 Proceedings IEEE, 2012, pp. 469-477. doi:10.1109/INFCOM.2012. 6195786 

of handset-based service usages, EPJ Data Science 1 (2012) 1-18. doi: 10.1140/epjds10.

[28] S. Isaacman, R. Becker, R. Cáceres, S. Kobourov, M. Martonosi, J. Rowland, A. Varshavsky, Identifying important places in peopleś lives from cellular network data, in: Pervasive computing, Springer, 2011, pp. 133151.

[29] J. A. Hartigan, Clustering, Annual review of biophysics and bioengineering 2 (1) (1973) 81-102.

[30] J. H. Friedman, Greedy function approximation: a gradient boosting machine, Annals of statistics (2001) 1189-1232.

[31] J. Friedman, T. Hastie, R. Tibshirani, The elements of statistical learning, Vol. 1, Springer series in statistics New York, 2001.

[32] Scikit-learn, Ensemble methods, http://scikit-learn.org/stable/ modules/ensemble.html, accessed: 2017-12-20. 